\title{
Investigation of Nonequilibrium Electronic Dynamics of Warm Dense Copper with Femtosecond X-Ray Absorption Spectroscopy
}

\author{
Jong-Won Lee $\odot,{ }^{1,2, \uparrow}$ Minju Kim $\odot,{ }^{1,2, \dagger}$ Gyeongbo Kang, ${ }^{1,2}$ Sam M. Vinko, ${ }^{3,4}$ Leejin Bae, ${ }^{5}$ Min Sang Cho $\odot,{ }^{1,2}$ \\ Hyun-Kyung Chung, ${ }^{6}$ Minseok Kim, ${ }^{7}$ Soonnam Kwon $\odot,{ }^{7}$ Gyusang Lee, ${ }^{1,2}$ Chang Hee Nam, ${ }^{1,2}$ Sang Han Park $\odot,{ }^{7}$ \\ Jang Hyeob Sohn, ${ }^{2}$ Seong Hyeok Yang, ${ }^{2}$ Ulf Zastrau, ${ }^{8}$ and Byoung Ick Cho $\oplus^{1,2, *}$ \\ ${ }^{1}$ Center for Relativistic Laser Science, Institute for Basic Science, Gwangju 61005, Republic of Korea \\ ${ }^{2}$ Department of Physics and Photon Science, Gwangju Institute of Science and Technology, Gwangju 61005, Republic of Korea \\ ${ }^{3}$ Department of Physics, University of Oxford, Parks Road, Oxford OXI 3PU, United Kingdom \\ ${ }^{4}$ Central Laser Facility, STFC Rutherford Appleton Laboratory, Didcot OX11 OQX, United Kingdom \\ ${ }^{5}$ Korea Atomic Energy Research Institute, Daejeon 34057, Republic of Korea \\ ${ }^{6}$ Korea Institute of Fusion Energy, Daejeon 34133, Republic of Korea \\ ${ }^{7}$ Pohang Accelerator Laboratory, Pohang, Gyeongbuk 37673, Republic of Korea \\ ${ }^{8}$ European XFEL Gmbh, Notkestrasse 85, D-22607 Hamburg, Germany
}

(Received 1 June 2021; revised 11 August 2021; accepted 23 September 2021; published 22 October 2021)

\begin{abstract}
Ultrafast optical excitation of matter leads to highly excited states that are far from equilibrium. In this study, femtosecond x-ray absorption spectroscopy was used to visualize the ultrafast dynamics in photoexcited warm dense $\mathrm{Cu}$. The rich dynamical features related to $d$ vacancies are observed on femtosecond timescales. Despite the success in explaining x-ray absorption data in the picosecond regime, the new femtosecond data are poorly understood through the traditional two-temperature model based on the fast thermalization concept and the static electronic structure for high-temperature metals. An improved understanding can be achieved by including the recombination dynamics of nonthermal electrons and changes in the screening of the excited $d$ block. The population balance between the $4 s p$ and $3 d$ bands is mainly determined by the recombination rate of nonthermal electrons, and the underpopulated $3 d$ block is initially strongly downshifted and recovered in several hundreds of femtoseconds.
\end{abstract}

DOI: 10.1103/PhysRevLett.127.175003

Recent advances in intense ultrafast lasers have enabled the creation of highly excited states of matter that are far from thermal equilibrium. The ultrafast absorption of energy in condensed matter results in extreme material conditions, known as warm dense matter (WDM), in which the thermal energy is comparable to the Fermi energy and the ions are strongly coupled [1,2]. WDM bridges the gap between condensed matter and plasma physics. Improving the fundamental understanding of ultrafast energy relaxation and the structural evolution in strongly out-of-equilibrium conditions is relevant for a wide range of fields, such as femtosecond laser ablation, micromachining, and even nanosurgery [3-5].

In terms of the response of systems irradiated by femtosecond laser pulses, the generally accepted concept is that an optical pulse directly excites electrons, which quickly thermalize, and that a finite electronic temperature is established in a few tens of femtoseconds $[6,7]$ while the lattice remains cold. Then, the two subsystems equilibrate through electron-phonon coupling. This is the basic premise underlying the two-temperature model (TTM) [8]. This framework has been extensively applied to calculate the optical and thermophysical properties of laser-irradiated matter and to develop advanced models for thermal and nonthermal melting [9-12], as well as to interpret data from various experiments involving the interaction of matter with ultrashort laser pulses [13-17].

However, the detailed dynamics of the electronic system might be more complicated than those described by the simple TTM. The hardening of phonon modes in noble metals is one of the interesting predictions in the highly excited two-temperature system $[18,19]$. The excitation of localized $d$ electrons may reduce the screening of the electron-ion potential. As a result, the $d$ band may shift toward lower energies and the melting temperature increases. However, to the best of our knowledge, a direct measurement of modified electronic structure in such systems has not been reported to date.

Recent experiments have reported the existence of longlived nonthermal electrons (NTe) $[20,21]$ and flux-limited ballistic transport by NTe [22] in photoexcited metals. Both cases entailed optical reflectivity measurements with femtosecond lasers which led to the conclusion that the simple high temperature electron distribution may not be sufficient to describe the femtosecond optical properties of highly excited systems properly. However, detailed information on thermal and nonthermal electrons in a broad energy range of free and localized states has yet to be investigated. 
In this study, a femtosecond measurement of the x-ray absorption of $\mathrm{WDCu}$ is presented. Time-resolved $\mathrm{x}$-ray absorption spectroscopy (TR-XAS) experiments demonstrated the capability to access the excited electron-hole distributions in broad energy ranges [23-27]. Upon exposure to femtosecond laser irradiation beyond the melting threshold, significant portions of localized $d$ electrons are excited, and the strongly perturbed sample evolves into $\mathrm{WDM}$ at a few eV temperatures. The evolution of unoccupied states in the $d$ band is probed by the absorption of femtosecond $\mathrm{x}$-ray pulses. The initially predicted $\mathrm{x}$-ray absorption based on the simple TTM and the static electronic density of states (DOS) of high-temperature liquid $\mathrm{Cu}$ revealed the limit of the traditional description of the femtosecond electronic structure responses. Instead, we calculated the dynamic shift of the excited $d$ band and also included the recombination dynamics of NTe in the TTM. These modifications can successfully reproduce the key features observed in the measurement and reveal the ultrafast population balance between conduction and localized electrons. This study provides evidence for the decade-old prediction of the more-localized $d$ states in excited noble metals and sheds light on improving our understanding of the material properties at the nascent stage of intensively photoinduced systems and phase transition dynamics.

The experiment was performed at the soft $\mathrm{x}$-ray scattering and spectroscopy station in the Pohang accelerator laboratory $\mathrm{x}$-ray free electron laser (PAL-XFEL) [28-30]. We employed the fs-XAS technique described in Ref. [31]. A 40 fs, $400 \mathrm{~nm}(3.1 \mathrm{eV})$ laser pulse was focused to a $150 \mu \mathrm{m}$ FWHM diameter on the sample. The incident fluence was $0.33 \pm 0.03 \mathrm{~J} / \mathrm{cm}^{2}$. The sample was $73 \pm 5 \mathrm{~nm}$ of polycrystalline $\mathrm{Cu}$ foil, coated on both sides with $100 \mathrm{~nm}$ of $\mathrm{SiO}_{2}$ layers. These were to prevent oxidation and delay the hydrodynamic expansion. Independently measured absorption by a $\mathrm{SiO}_{2}$ layer was less than $3 \%$. With measured laser absorption of approximately $65 \%$, the deposited energy density, $E_{d}$ was $3.4 \pm 0.34 \mathrm{MJ} / \mathrm{kg}$. Owing to the high laser fluence, the sample was translated to a fresh area after the irradiation of each pulse.

Figure 1 shows conceptual sketches of the very early stage of electron distribution in the photoexcited $\mathrm{Cu}$. Under ambient conditions, the $3 d$ band is fully occupied, and its top edge was approximately $2 \mathrm{eV}$ below the Fermi level, $E_{F}$. The photons $(3.1 \mathrm{eV})$ are absorbed through the $3 d \rightarrow 4 p$ transition, and a strong nonequilibrium condition is created by the end of the laser pulse duration. Eventually, all electrons are fully thermalized to form a Fermi distribution with a finite $T_{e}$ and further equilibrated with the cold lattice through electron-phonon coupling in the order of $1-10 \mathrm{ps}[7,10,13]$.

XFEL pulses with $50 \mathrm{fs}$ duration were used as a probe of the unoccupied $d$ states between Figs. 1(b) and 1(c). The XFEL photon energy varied between 928.5 and $930.6 \mathrm{eV}$ with approximately $1 \mathrm{eV}$ bandwidth to cover the pre- $L$-edge
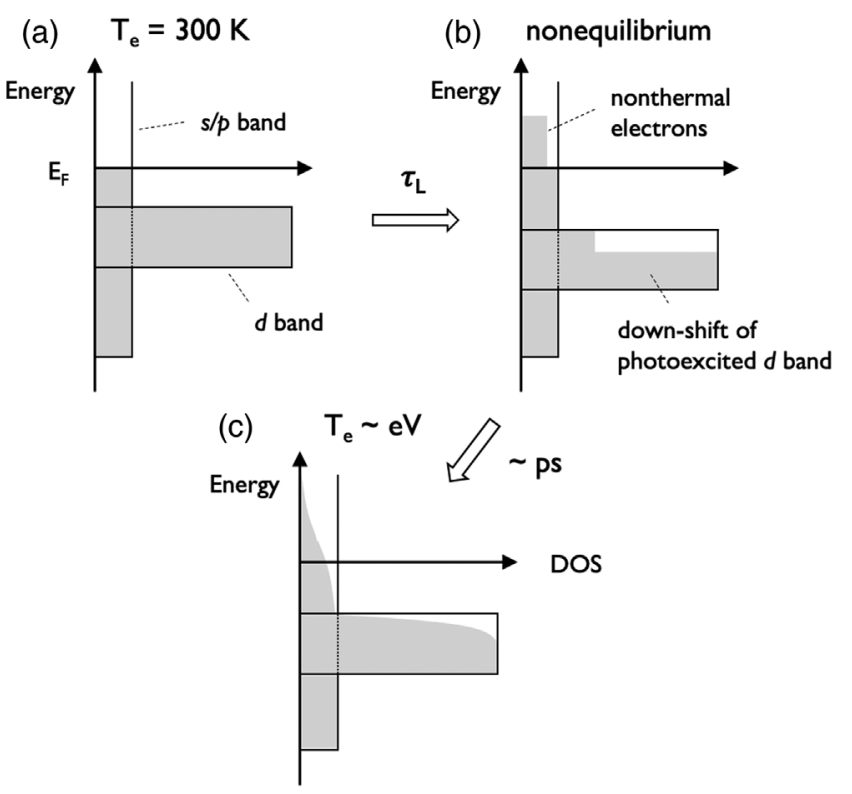

FIG. 1. Sketches of femtosecond laser excited electronic system of noble metal at distinguished phases. (a) Cold $\mathrm{Cu}$ before heating. The $d$ band is fully occupied. (b) Just after laser irradiation ( $\tau_{L}$ : pulse duration). The depopulated $d$ band is downshifted, and NTes are generated above the $E_{F}$. (c) In a few picoseconds, electrons have equilibrated forming a high temperature Fermi distribution. The evolution of unoccupied $d$ states between (b) and (c) are probed through the absorption of the XFEL pulse.

XAS regime of cold $\mathrm{Cu}$. Through the $2 p \rightarrow 3 d$ transitions, the $x$-ray absorption was sensitive to the evolution of the hole distribution near the top edge of the $d$ band. The absorption of a single XFEL pulse was determined by the simultaneous measurement of the incident and transmitted pulse energies. Including the timing jitter between the optical and XFEL pulses, the temporal resolution was $200 \mathrm{fs}$. More details on the beam line and diagnostics are provided in Refs. [29-31].

Figure 2 shows the measured pre- $L$-edge absorption at the selected pump-probe delays. Each data point is an average of 20 measurements. At $0 \mathrm{fs}$, the flat and low absorption indicates that the $d$ band is fully occupied before optical pumping. At $200 \mathrm{fs}$, the strong enhancement is observed around $929.5 \mathrm{eV}$, which corresponds to $-3 \mathrm{eV}$ below the $E_{F}$. At $500 \mathrm{fs}$, the peak shifts to around $930.1 \mathrm{eV}$ and remains visible until $1600 \mathrm{fs}$, while the overall absorption decreases. After 2 ps, the absorption decreases monotonically with decreasing photon energy.

As the first attempt to interpret the data, XAS calculations were performed following the method used in previous picosecond studies [23,33]. For an average $E_{d}$ of $3.4 \mathrm{MJ} / \mathrm{kg}$, time-dependent $T_{e}(t)$ was calculated using the following TTM equations:

$$
C_{e}\left(T_{e}\right) \frac{\partial T_{e}(t)}{\partial t}=-G\left(T_{e}\right)\left[T_{e}(t)-T_{l}(t)\right]+S_{L}(t)
$$




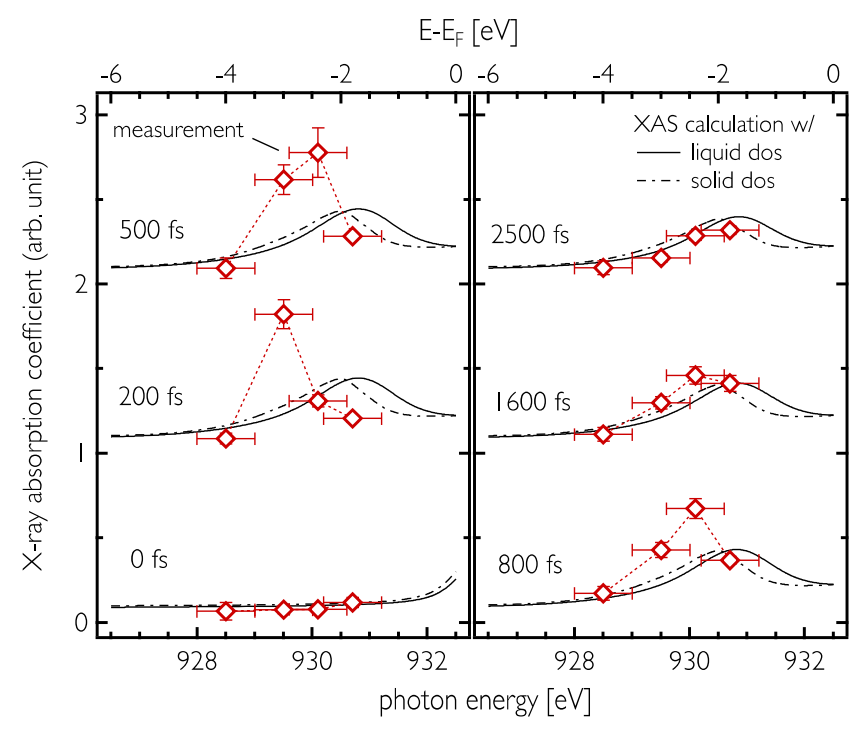

FIG. 2. Pre- $L$-edge XAS of the $\mathrm{Cu} d$ band at selected pumpprobe delays. Measurements are compared with the calculated high temperature absorption based on the TTM and the static DOSs. Spectra are displayed with an offset of +1 along the vertical axis between time steps.

$$
C_{l} \frac{\partial T_{l}(t)}{\partial t}=G\left(T_{e}\right)\left[T_{e}(t)-T_{l}(t)\right]
$$

The subscripts $e$ and $l$ represent the electron and lattice, respectively. $T, C$, and $G$ are the temperature, heat capacity, and electron-phonon coupling parameter, respectively. $S_{L}$ represents the deposited $E_{d}$ by the laser. The $C_{l}$ is $3.5 \times 10^{6} \mathrm{~J} / \mathrm{m}^{3} \mathrm{~K}$, obtained by the Dulong-Petit law. In the range of $300-1300 \mathrm{~K}$, the heat capacity of $\mathrm{Cu}$ at a constant volume increases by about $10 \%$, and is so assumed to be constant [34].

The absorption at each delay was calculated by applying a high temperature hole distribution on both liquid and solid DOSs from Ref. [33]. For easy reading of the graph, the experimental errors were not included in the calculation curves. With the experimental resolution of $1 \mathrm{eV}$, the difference in XAS originating from the different DOSs was not significant. At all delays, the calculation predicted absorption peaks at approximately 930.5-931 eV. Interestingly, these calculations failed to reproduce most of the measurements in 200-1600 fs. A reasonable agreement is observed only after $2 \mathrm{ps}$, which is consistent with the previous picosecond study [23,33]. This implies that the simple TTM based on the instant electron thermalization concept and the static electronic structure at high temperatures may not be sufficient to describe the initial response of the electronic structure to intense femtosecond laser pulses.

To improve our understanding, we additionally considered two dynamic procedures, that is, the shift of the excited $d$ band and the decay of NTe. We first calculated the DOSs for heated $\mathrm{Cu}$ using finite-temperature density

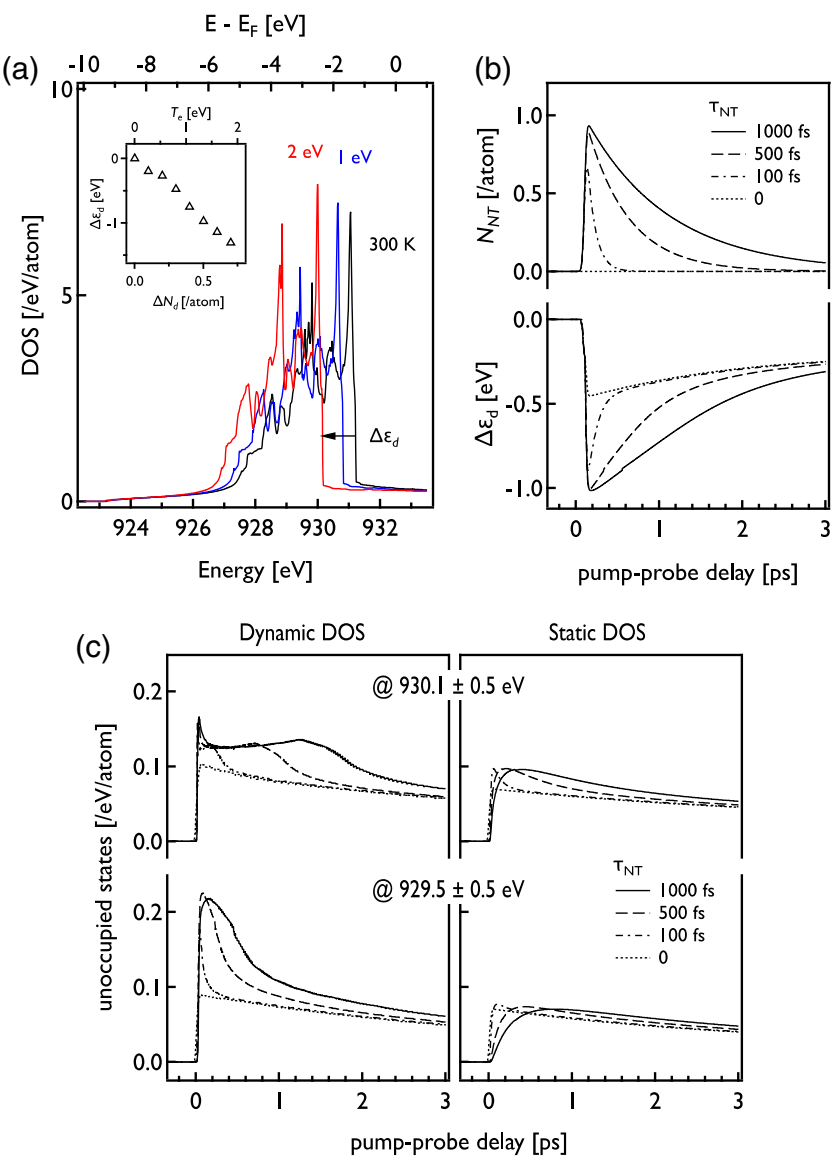

FIG. 3. (a) ABINIT calculation of DOS of $\mathrm{Cu}$ at $300 \mathrm{~K}, 1 \mathrm{eV}$, and $2 \mathrm{eV}$. (inset) The shift of $d$ block as a function of $d$-electron excitation. (b) Evolutions of NTe density and the $d$-band shift with various NTe lifetimes. (c) The evolution of hole densities at 930.1 and $929.5 \mathrm{eV}$ in dynamic and static DOSs.

functional theory using the ABINIT code [35-37]. We assumed an FCC $\mathrm{Cu}$ lattice with valence electrons at temperatures between $300 \mathrm{~K}$ and $2 \mathrm{eV}$. The electronic structure was calculated using projector augmented wave pseudopotentials for the core states with a frozen Ne core, and employed exchange and correlation potentials in the Perdew, Burke, and Ernzerhof form of the generalized gradient approximation [38]. The simulations used a $20 \times 20 \times 20$ Monkhorst-Pack grid [39] with $256 k$ points to sample the Brillouin zone, a plane wave cutoff of up to $\sim 1000 \mathrm{eV}$, and up to 200 bands. The tetrahedron method was used for Brillouin zone integration to extract the DOS.

Figure 3(a) shows some examples of the calculated DOS at different temperatures. At $T_{e}=2 \mathrm{eV}$, the top edge of the $d$ band is downshifted by approximately $1.3 \mathrm{eV}$ owing to the depopulation of $d$ electrons, $\Delta N_{d}$. In the inset, the shift of the $d$ band, $\Delta \varepsilon_{d}$, is observed as a function of $T_{e}$, as well as the corresponding $\Delta N_{d}$. The $d$ block monotonically shifted to lower energies with increasing vacancies. These results are also consistent with the early calculations by Recoules et al. [18,19]. 
Next, the 11 electrons in the $3 d$ and $4 s p$ orbitals are divided into two groups, that is, nonthermal and thermal electrons. While the evolution of the total energy in the electronic system is described by Eq. (1), the NTe density is described separately by the following equation:

$$
\frac{\partial N_{n t}(t)}{\partial t}=-\frac{N_{n t}(t)}{\tau_{n t}}+N_{\mathrm{ph}}(t)
$$

where $N_{n t}$ and $\tau_{n t}$ denote the density and lifetime of the NTe, respectively. $N_{\text {ph }}=S_{L} / h \nu_{L}$ denotes the absorbed photon number density. Previous research on the selfreflectivity of WDAu irradiated with femtosecond laser pulses indicated the hot electron number at the end of laser pulse is about the same as the absorbed photon number [21]. Therefore, it is also assumed a single NTe in the conduction band is produced as a result of single-photon absorption. The detailed decay procedures of $d$ holes, such as the cascade Auger processes, are not considered because the typical $d$ hole lifetime is known to be sub 10 fs [6], which is much faster than the experimental resolution of this study. The excited electrons in the conduction band rapidly accumulate near the $E_{F}$ [32]. Therefore, for simplicity, NTe are assumed to be monoenergetic. The rest of the $N_{\text {th }}=11-N_{n t}$ electrons are considered to form a Fermi distribution with $T_{e, \text { th }}$, but different from $T_{e}$ in Eq. (1). $T_{e, \text { th }}$ is determined from the thermal electron energy obtained by subtracting the NTe energy from the total electron energy.

Figure 3(b) shows the evolution of $N_{n t}$ with several $\tau_{n t}$, and the corresponding shift of the $d$ band. In the case of no $\mathrm{NTe}\left(\tau_{n t}=0 \mathrm{fs}\right), 11$ electrons are instantly thermalized. At the $E_{d}$ of $3.4 \mathrm{MJ} / \mathrm{kg}$, the $d$ band is expected to be shifted by approximately $0.5 \mathrm{eV}$ at the end of laser pulse, and restored as $T_{e}$ decrease owing to the electron-lattice coupling. In contrast, with a finite $\tau_{n t}$, the conduction band was more populated and the $d$ band was less populated, that is, $\Delta N_{d}$ was greater than the $\tau_{n t}=0$ case. Then, using the inset of Fig. 3(a), the DOS with the same $d$ vacancy and the corresponding band shift were determined. Initially, the $d$ block was shifted by $>1 \mathrm{eV}$. Eventually, it was restored to the $\tau_{n t}=0$ case, however the governing timescale is not determined by the electron-phonon coupling but the NTe lifetime.

Based on the dynamic response of the $d$ band, timedependent unoccupied $d$ states at the probe energies of $929.5 \pm 0.5 \mathrm{eV}$, and $930.1 \pm 0.5 \mathrm{eV}$ are calculated [Fig. 3(c)]. For comparison, the same calculations with the static DOS are also shown. With $\tau_{n t=} 0$, the differences were not significant because the holes mainly accumulated near the top edge $d$ band (approximately $931 \mathrm{eV}$ ), which is 1-1.5 eV higher than the probe window. Even with the dynamic $d$ band, $\Delta \varepsilon_{d}$ is still less than $0.5 \mathrm{eV}$, and the effect is not significant.

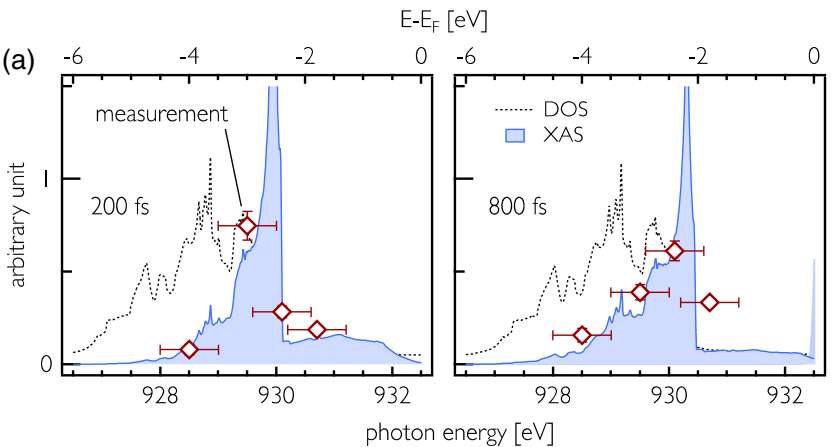

(b)

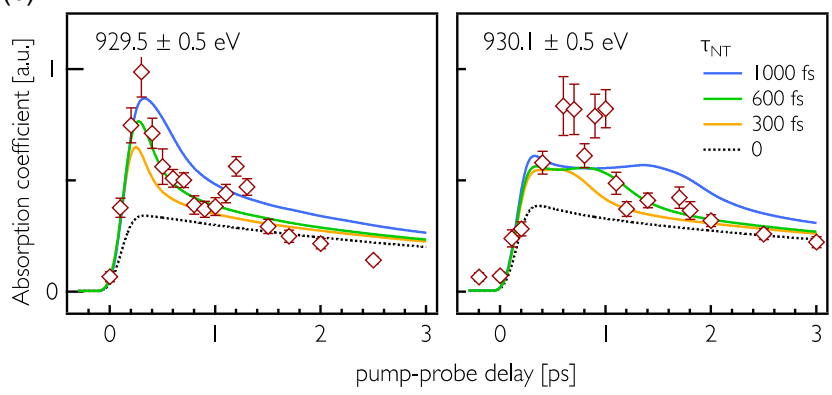

FIG. 4. (a) Measured absorptions (red diamonds) at 200 and $800 \mathrm{fs}$ are compared again with the dynamic DOS (dot line) and absorption calculation including NTe $\left(\tau_{n t}=500 \mathrm{fs}\right)$ and dynamic DOS (blue). (b) Evolution of x-ray absorption at 929.5 and $930.1 \mathrm{eV}$. Experiments are compared with calculations with various $\tau_{n t}$.

In contrast, with a finite $\tau_{n t}$ and dynamic DOS, the initial $\Delta \varepsilon_{d}$ exceeded $1 \mathrm{eV}$. Now, the top edge of the $d$ band initially falls into the probe range of $929.5 \pm 0.5 \mathrm{eV}$, and a sharp increase of hole density is expected. It decreases as NTe decay, and the top edge moves toward higher energy. In $930.1 \pm 0.5 \mathrm{eV}$, the hole density remained relatively flat until the top edge completely moved out of the range. The length of the plateau was roughly proportional to $\tau_{n t}$. It is also noted that if the $d$ band was static and NTe had a long lifetime, the unoccupied states in the probe windows increased slowly. In this case, while many energetic NTe existed in the early period, the rest of the electrons had a relatively lower temperature. As the NTe decayed, the $d$ band was heated slowly. Overall, at both probe energies, more unoccupied states and rich dynamical features were expected.

Finally, the x-ray absorptions were recalculated, including both dynamic DOS and NTe, and compared again with the experiment [Fig. 4]. $\tau_{n t}$ was set to 500 fs. To exhibit the detailed shift of the hole distribution and absorption peak, the experimental resolution was not convolved. As discussed earlier, at $200 \mathrm{fs}$, the top edge of the redshifted $d$ band and the absorption peak fell into the probing window of $929.5 \pm 0.5 \mathrm{eV}$. Later at $800 \mathrm{fs}$, as the $d$ band was restored, the calculated absorption peak also shifted to higher energies. These essentially reproduced the major experimental observations prior to $1 \mathrm{ps}$. 
More detailed comparisons at 929.5 and $930.1 \mathrm{eV}$ with different $\tau_{n t}$ are also presented in Fig. 4(b). The calculated curves are convolved with the temporal resolution of the experiment. At $929.5 \mathrm{eV}$, the measured absorption peaks at approximately the end of the optical laser, and decays rapidly. This can be explained by the recombination of NTe in a few $100 \mathrm{fs}$. At $930.1 \mathrm{eV}$, the most notable feature from the experiment is an enhanced, however relatively unchanged absorption between 500 and 1000 fs, although some fine structures may exist. This corresponds to the plateau structure in Fig. 3(c) and is also reproduced in the absorption calculations. Here, we do not attempt to fit the experimental data, however $\tau_{n t}=500-600$ fs provides reasonable agreement for both energies. According to the Fermi-liquid theory and two-photon photoemission experiments on $\mathrm{Cu}$, the relaxation time of electrons at the energy state $E=E_{F}+$ $0.5 \mathrm{eV}$ is about $100 \mathrm{fs}$ [32]. Therefore 500-600 fs timescales indicate that the NTe are mainly within a few hundreds $\mathrm{meV}$ range above the $E_{F}$. It is noted that some discrepancies infer that there may be additional effects not included in the simple model. For example, the secondary peak at $929.5 \mathrm{eV}$ and some fine structures at $930.1 \mathrm{eV}$. Possible reasons could be the structural change and its effect on the relaxation processes. These can be further investigated with improved temporal and spectral resolutions.

In this study, the ultrafast adjustment of the excited $d$ band in WDCu is investigated through the femtosecond XAS technique. Absorption measurement of the XFEL pulse for $\mathrm{Cu}$ nanofoil irradiated by an intense femtosecond laser shows a strongly enhanced overall absorption by $d$ states, the emergence of an absorption peak at the unexpected energy, and its fast shift in $0-2$ ps. These rich dynamical features related to localized states in WDM have not been observed in earlier investigations with picosecond resolutions, nor could they be explained by the traditional TTM and the static electronic structure calculation for hightemperature liquid metal. The theoretical description can be improved with two additional dynamic procedures. A simple kinetic model for NTe in the conduction band was considered in addition to the TTM. In the first few ps, the population balance between the $4 s p$ and $3 d$ bands is mainly determined by the recombination rate of $\mathrm{NTe}$ instead of electron-phonon coupling. The electronic DOS for heated $\mathrm{Cu}$ is recalculated with the ABINIT code, which includes changes in the screening of excited localized electrons. Combined with the NTe kinetics, the initial downshift of the excited $d$ block and its recovery can be determined. These modifications successfully reproduced the key features observed in femtosecond $\mathrm{x}$-ray absorption measurements. This investigation provides evidence for a decade-old prediction of the shift of the $d$ band of noble metals under intense laser irradiation, and sheds light on further improving our understanding of the ultrafast excitation and phase transition dynamics. Fundamental and interesting questions, such as the lattice stability of metals undergoes femtosecond laser melting, and the nonequilibrium thermophysical properties could be further studied.

We acknowledge the technical support provided by the PAL-XFEL. This work was supported by the Institute for Basic Science (IBS-R012-D1), the National Research Foundation of Korea (NRF-2015R1A5A1009962, NRF2019R1A2C2002864, NRF-2020K1A3A7A09080397), and GRI grant funded by the GIST in 2021. This study was also partly supported by NRF grants (NRF2018R1D1A1B07046676, NRF-2020R1A2C1007416). S. M. V. acknowledges support as a Research Fellow of the Royal Society, and further acknowledges support from the U.K. EPSRC under Grant No. EP/P015794/1.

*Corresponding author.

bicho@gist.ac.kr

These authors contributed equally to this work.

${ }^{\ddagger}$ Present address: Pohang Accelerator Laboratory, Pohang, Gyeongbuk 37673, Republic of Korea.

[1] S. Ichimaru, Rev. Mod. Phys. 54, 1017 (1982).

[2] R. W. Lee et al., Laser Part. Beams 20, 527 (2002).

[3] R. R. Gattass and E. Mazur, Nat. Photonics 2, 219 (2008).

[4] Q. Feng, Y. N. Picard, H. Liu, S. M. Yalisove, G. Mourou, and T. M. Pollock, Scr. Mater. 53, 511 (2005).

[5] A. Vogel, J. Noack, G. Hüttman, and G. Paltauf, Appl. Phys. B 81, 1015 (2005).

[6] B. Y. Mueller and B. Rethfeld, Phys. Rev. B 87, 035139(2013).

[7] J. Winter, S. Rapp, M. Schmidt, and H. P. Huber, Appl. Surf. Sci. 417, 2 (2017).

[8] S. Anisimov, B. Kapeliovich, and T. Perelman, Zh. Eksp. Teor. Fiz. 66, 375 (1974), http://www.jetp.ras.ru/cgi-bin/dn/ e_039_02_0375.pdf.

[9] S. Mazevet, J. Clérouin, V. Recoules, P. M. Anglade, and G. Zerah, Phys. Rev. Lett. 95, 085002 (2005).

[10] Z. Lin, L. V. Zhigilei, and V. Celli, Phys. Rev. B 77, 075133 (2008).

[11] O. Lozan, R. Sundararaman, B. Ea-Kim, J.-M. Rampnoux, P. Narang, S. Dilhaire, and P. Lalanne, Nat. Commun. 8, 1656 (2017).

[12] Y. Dubi and Y. Sivan, Light Sci. Appl. 8, 89 (2019).

[13] J. Hohlfeld, S. S. Wellershoff, J. Güdde, U. Conrad, V. Jähnke, and E. Matthias, Chem. Phys. 251, 237 (2000).

[14] R. Ernstorfer, M. Harb, C. T. Hebeisen, G. Sciaini, T. Dartigalongue, and R. J. D. Miller, Science 323, 1033 (2009).

[15] Z. Chen, V. Sametoglu, Y. Y. Tsui, T. Ao, and A. Ng, Phys. Rev. Lett. 108, 165001 (2012).

[16] M. Z. Mo et al., Science 360, 1451 (2018).

[17] S. M. Vinko, V. Vozda, J. Andreasson, S. Bajt, J. Bielecki et al., Phys. Rev. Lett. 124, 225002 (2020).

[18] V. Recoules, J. Clérouin, G. Zérah, P. M. Anglade, and S. Mazevet, Phys. Rev. Lett. 96, 055503 (2006).

[19] E. Bévillon, J.-P. Colombier, V. Recoules, and R. Stoian, Phys. Rev. B 89, 115117 (2014).

[20] F. Bisio, E. Principi, M. Magnozzi, A. Simoncig, E. Giangrisostomi, R. Mincigrucci, L. Pasquali, C. Masciovecchio, F. Boscherini, and M. Canepa, Phys. Rev. B 96, 081119(R) (2017). 
[21] M. Kim, J. H. Jung, S. H. Yang, M. S. Cho, G. Kang, G. Lee, J.-W. Lee, S. Lee, J. Sohn, and B. I. Cho, Appl. Surf. Sci. 561, 150073 (2021).

[22] Z. Chen, V. Sametoglu, Y. Y. Tsui, T. Ao, and A. Ng, Phys. Rev. Lett. 108, 165001 (2012).

[23] B. I. Cho et al., Phys. Rev. Lett. 106, 167601 (2011).

[24] J. Gaudin et al., Sci. Rep. 4, 4724 (2014).

[25] K. Engelhorn, V. Recoules, B. I. Cho, B. Barbrel, S. Mazevet, D. M. Krol, R. W. Falcone, and P. A. Heimann, Phys. Rev. B 91, 214305 (2015).

[26] A. Fernandez-Pañella et al., Phys. Rev. B 101, 184309 (2020).

[27] N. Jourdain, L. Lecherbourg, V. Recoules, P. Renaudin, and F. Dorchies, Phys. Rev. Lett. 126, 065001 (2021).

[28] H.-S. Kang et al., Nat. Photonics 11, 708 (2017).

[29] S. H. Park et al., Rev. Sci. Instrum. 89, 055105 (2018).

[30] M. Kim, C.-K. Min, and I. Eom, J. Synchrotron Radiat. 26, 868 (2019).
[31] J.-W. Lee, G. Kang, M. Kim, S. Park, S. Kwon, S. Yang, and B. Cho, J. Synchrotron Radiat. 27, 953 (2020).

[32] E. Knoesel, A. Hotzel, and M. Wolf, Phys. Rev. B 57, 12812 (1998).

[33] B. I. Cho, T. Ogitsu, K. Engelhorn, A. A. Correa, Y. Ping, J. W. Lee, L. J. Bae, D. Prendergast, R. W. Falcone, and P. A. Heimann, Sci. Rep. 6, 18843 (2016).

[34] G. K. White and S. J. Collocott, J. Phys. Chem. Ref. Data 13, 1251 (1984).

[35] M. Torrent, F. Jollet, F. Bottin, G. Zérah, and X. Gonze, Comput. Mater. Sci. 42, 337 (2008).

[36] X. Gonze et al., Comput. Phys. Commun. 180, 2582 (2009).

[37] X. Gonze et al., Comput. Phys. Commun. 205, 106 (2016).

[38] J. P. Perdew, K. Burke, and M. Ernzerhof, Phys. Rev. Lett. 77, 3865 (1996).

[39] H. J. Monkhorst and J. D. Pack, Phys. Rev. B 13, 5188 (1976). 